\title{
Personality, Gender, and Age as Predictors of Media Richness Preference
}

\author{
David R. Dunaetz, ${ }^{1}$ Timothy C. Lisk, ${ }^{2}$ and Matthew Minsuk Shin ${ }^{3}$ \\ ${ }^{1}$ Department of Leadership and Organizational Psychology, Azusa Pacific University, Azusa, CA 91702, USA \\ ${ }^{2}$ Cornerstone On Demand, San Francisco, CA 94107, USA \\ ${ }^{3}$ Department of International Trade, College of Commerce \& Economics, Konkuk University, Sanghuh Hall No. 638, \\ 120 Neungdong-ro, Kwangjin-gu, Seoul 143-701, Republic of Korea
}

Correspondence should be addressed to Matthew Minsuk Shin; shinm@konkuk.ac.kr

Received 29 April 2015; Accepted 22 July 2015

Academic Editor: Constantine Kotropoulos

Copyright (C 2015 David R. Dunaetz et al. This is an open access article distributed under the Creative Commons Attribution License, which permits unrestricted use, distribution, and reproduction in any medium, provided the original work is properly cited.

Media richness, the degree to which a specific media transmits information in multiple channels, is an important concept as the number of available multimedia communication methods increases regularly. Individuals differ in their preferences for media richness which may influence their choice of communication multimedia in a given situation. These preferences can influence how successful their communication efforts will be. This exploratory study of 299 adults (ages 16-84) with at least a basic ability to compute examines the relationship between multimedia preference and age, gender, and personality traits. Males and people with higher levels of extraversion and agreeableness were found to have a higher preference for media richness. Age was not a significant predictor of media richness preference.

\section{Introduction}

As the number of media available for interpersonal communication increases, the decision of what medium to use in a given context becomes more difficult. Although the context of the communication will have the greatest influence on the choice of media, individual differences, such as age, gender, and personality traits of those who communicate, can also play a role. Some people may have a strong preference for richer media like face-to-face interaction or the telephone, media which allow the message to be transmitted in multiple ways (e.g., word choice and tone of voice, as well as facial expressions and other forms of nonverbal communication) and which allow for immediate reception of the message, immediate feedback that the message has been received, and immediate elaboration or clarification when necessary $[1,2]$. Other people may tend towards leaner media, such as electronic communication (e.g., email and text messaging), which primarily use written text and for which longer delays in response are expected before feedback is given to the sender. Because of these individual differences, attempts at communication might be more effective if the transmitter of the message is aware of the perceiver's preferences. The relationships between individual differences and preference for different media are not clearly understood, thus making choosing the most appropriate media more difficult. In order to make better decisions concerning media choice, this novel, exploratory study examines how preference for media richness is related to personality, age, and gender.

\section{Literature Review}

When a sender chooses a medium for transmitting information to another person, he or she must consider a multitude of factors. These factors include the characteristics of the available media, environmental characteristics (such as the nature of the message and social factors), and the personal qualities of both the sender and the receiver. An inappropriate 
TABLE 1: Media richness of various media.

\begin{tabular}{lccc}
\hline Media & Synchronicity & Number of cues & Ordinal ranking of media richness (1: low; 5: high) \\
\hline (1) Face-to-face & High & High & 5 \\
(2) Telephone & High & Medium & 4 \\
(3) Instant messaging & Medium-high & Low & 3 \\
(4) Text messaging & Medium & Low & 2 \\
(5) Email & Low & Low & 1 \\
\hline
\end{tabular}

choice of media can result in poor or incomplete transmission and comprehension of the intended message.

2.1. Media Richness. Daft and Lengel $[1,2]$ provide a framework for understanding one aspect of a communication medium. Media richness is a measure of a medium's ability to transmit information that will change the receptor's understanding within a given time. They theorize that four measures determine the richness of a medium: (1) the delay necessary for providing feedback after reception of the message (richer media have shorter delays; they are higher in synchronicity), (2) the number of cues that are used to transmit information (e.g., face-to-face communication uses spoken words, tone of voice, facial expressions, and body language to transmit the message; text messages are usually limited to written text and emoticons), (3) the degree of personalization of a message (a phone call is more personal than a radio advertisement), and (4) the freedom that the media gives to choose a variety of styles of language (a person can use a great variety of language styles on the phone or in email; an organization's formal written policies are more limited in style).

In this study, we focus on interpersonal communication, so we examine media that are used in communication between individuals (i.e., not one-to-many communication or mass-media). The five we have chosen to examine represent a broad mixture of traditional and modern multimedia communications: face-to-face communication, telephone, email, text messaging (also known as SMS, short message service, or simply as texting), and instant messaging (also known as IM or chatting). Many other media exist that can be used for interpersonal communication (e.g., letters, memos, video conferencing, Facebook, Twitter, and Pinterest, with new media created regularly). In interpersonal communication, there is no limit to the style of language that can be used. By definition, all interpersonal communication is able to be personalized. This means that these two dimensions of media richness (choices of language styles and degree of personalization) are not relevant to the media in this study. Thus the only dimensions of media richness in Daft and Lengel's framework $[1,2]$ which are relevant in this study are (1) immediacy of feedback (synchronicity) and (2) the number of cues used to transmit information. The capacity and expectation for immediate feedback are highest in face-toface communication and telephone, almost as high in instant messaging, medium in texting, and lowest in email [3]. The number of cues used to transmit information is highest in face-to-face communication, not quite as high in telephone, and lowest in IM, texting, and email $[3,4]$. Treating these two dimensions as being equally important yields the following order (Table 1), from richest to leanest media: face-to-face communication, telephone, instant messaging, texting, and email [5].

2.2. Environmental Factors. The choice of media in light of environmental factors such as the nature of the task and the social context has been the subject of much research. Many theories contribute to our understanding of how humans make this choice. Rational choice theory $[6,7]$ predicts that people choose a communication medium by matching its characteristics to requirements of the communication task. It would be a rational choice to send 25 pages of part numbers by email rather than communicate this information over the phone. In some situations, the nature of the task accounts for almost all the variance in the choice of the communication medium.

Media richness theory $[1,2]$ predicts that richer media are needed when there is greater ambiguity or uncertainty in the information being communicated. For example, complex problems should be discussed in person, rather than through a letter. The cognitive based view of media choice [8] is based on the elaboration likelihood model of attitude formation and change [9] which states that, in order to process a message, the recipient must be both motivated and able to do so. This theory proposes that people are able to process simple or small amounts of information in face-to-face communication, but complex or larger amounts of information would be more appropriate for email or other leaner media that do not require immediate feedback. Similarly, people are more motivated to process messages when another person is present (such as in rich media) than when the message is received apart from the presence of the sender. Thus social presence is a major environmental factor to consider when choosing a medium.

Other environmental factors related to media choice are also influenced by the social context [7, 10]. If the recipient of the message is not physically present, face-toface communication is not possible. If a person has not been in an environment where a certain medium has been used, choosing it is not a very likely option. However, social learning [11] occurs frequently when one observes peers using a new medium. If many of a person's friends are using a new means of communication, the person will be motivated to join in the communication and may learn how to use the medium through a combination of imitation and trial and error. The greater the enthusiasm for and meaning associated 
with a communication channel in a social context, the greater the motivation to choose that channel.

It has been postulated that rich media which allow for greater social contact between the two parties are better for building relationships (social presence theory [12]); face-toface communication would be the best for building relationships and leaner electronic media would be the least effective. But, as the Internet has developed, building relationships through lean media has become common and many people find it more effective than face-to-face communication for developing relationships. Walther's [13] social information processing perspective explains why computer mediated communication can sometimes be more effective in developing relationships. In computer mediated communication, as in all human interactions, participants are motivated to form relationships. They gather information and create hypotheses about the people with whom they communicate. Their interchange will confirm or modify these hypotheses. Thus, in lean media (with fewer cues or longer delays), relationship development will occur as with richer media but at a slower pace since less information is exchanged with possibly longer delays. It is quite possible that this type of relationship development is more attractive to some people than others. This provides one explanation of why individual differences also play a role in media choice.

2.3. Individual Differences. Whereas the environmental factors influencing media choices have been often studied, this exploratory study will examine some of the personal qualities of those involved in communication which might influence one's choice of media. These qualities might include abilities, motivation, demographics, cultural background, and personality traits. These qualities tend to be highly interrelated. For example, ability may be most directly influenced by education and social context, which in turn are influenced by demographics such as age, gender, and cultural background. Ability is also linked to values and motives. For example, a favorable attitude to change and innovation is a strong predictor of use of computer mediated communication [14].

The present study examines the predictive power of easily observable individual differences to predict motivation in media choice. Age and gender are among the most easily identifiable individual differences. Many personality traits are also easy to identify through regular conversation with a person [15]. Motives and preferences, however, are much harder to identify. Some motives are identifiable through selfreport and are relatively easy to identify (explicit motives), but others (implicit motives) are difficult to access and perhaps not even knowable $[16,17]$. Some people are likely to be more motivated to use richer media than others. We identify this motive as media richness preference. This study will examine the relationship between media richness preference and personality traits as described by the five-factor model of personality, in addition to gender and age.

The five-factor model of personality [18-20] is the most commonly used model of personality traits in modern research psychology. Traits may be considered recognizable and habitual patterns of behavior and sometimes include habitual patterns of thoughts and emotions. The five-factor model describes personality along five continuous trait dimensions (known as the Big Five): extraversion, agreeableness, conscientiousness, openness to experience, and neuroticism.

This exploratory study examines how these five dimensions of personality (as well as age and gender) are related to an individual's preference for using channels of communication that are richer relative to those which are leaner. Preferences are affective reactions to stimuli [21] and thus may be positive or negative and vary in intensity. In this study, we examine people's reaction to a communication medium by asking them about their preference for that medium in comparison to another medium that is either higher or lower in media richness. These affective reactions may have a cognitive basis to them (e.g., "I don't like the telephone because it's almost always my demanding motherin-law calling."), but often they have a purely evaluative content that precedes cognition [21, 22]. These evaluations serve as the basis for motivation, either as pure affect or in interaction with cognitions that may follow the perception of the stimulus. Thus we believe that these preferences will be related to the Big Five personality traits, as are other motives $[23,24]$. Since preference measures affect or motivation, being able to predict media richness preference from easily observable and measurable personality traits can lead to the practical application of this knowledge when choosing a communication medium to use with an individual.

Previous research has indicated that affect associated with a communication medium is a legitimate construct that can be reliably measured [25]. We do not try to measure affect directly for each of the media that are being considered, but we measure the preference for one medium relative to another. This is appropriate for comparing items that are ranked by an ordinal scale, such as the media richness of the media in question [26].

Another research has concerned the relationship between personality and other communication constructs. McCroskey et al. [27] found that personality traits predict shyness, sense of competence in communication, communication apprehension, verbal aggressiveness, communication compulsivity, and argumentativeness. Few studies have examined media richness preference and personality. D. J. Reid and F. J. M. Reid [28] found that people who preferred texting over telephone were higher in social anxiety, a construct associated with neuroticism. Karemaker [29] examined a similar comparison and found that extraversion predicted a preference for face-to-face over computer mediated communication, but only for those who were low in neuroticism. This exploratory study seeks to go beyond these media specific studies and explore whether personality predicts a general preference for media richness that is applicable across multiple means of communication.

\section{Hypotheses}

We examine whether individual differences (age, gender, and personality traits) predict preference for media richness 
in interpersonal communication among adults. As this is an exploratory study, our hypotheses are quite general and nondirectional in their predictions.

3.1. H1: Age Predicts Media Richness Preference. As younger people learn and adapt to new technologies faster than older people [30], it could be assumed that younger people will have a preference for leaner media. But if an older person only uses face-to-face interaction and telephone to communicate, the person would have a high preference for leaner media if he or she strongly preferred telephone to face-to-face communication, just as if a younger person used many media but tended to prefer email over other forms. We know of no developmental theory that would predict that younger or older people would have a higher or lower preference for media richness. We include this nondirectional hypothesis to discover any potential relationship that might warrant further study.

3.2. H2: Gender Will Predict Preference for Media Richness. Males and females have very different patterns of communication [31-33]. Men tend to be agentic or instrumental; their communication is more active, decisive, direct, and oriented towards obtaining results. Women tend to be communal or expressive; their communication is more expressive, caring, and oriented towards developing relationships. Women generally are more responsive and interested in communication than men [34]. They more often express their feelings while communicating [35]. They are less likely to dominate the communication process and more likely to let the other person express himself or herself freely [36]. Women use and understand nonverbal communication more than men [37] and work harder at interpreting it properly [38].

In general, it may be said that women are more skilled than men in communication [36-39]. But how would this affect media richness preference? It could be argued that females would prefer rich media because they get more out of all forms of communication. The additional richness would bring more benefits to them than to men. In other words, men would not profit as much from multiple cues as women do, so men would have a higher preference for lean media than women which would allow them more time to respond appropriately. This is similar to the social compensation hypothesis [40] which proposes that the socially anxious are more likely to profit from computer mediated communication because the communication environment is less threatening.

From an alternative point of view, it could also be argued that females have less of a need for media richness because their skills enable them to use all means effectively; the cues that are lost with the leaner media would have less impact on females than on males. Thus preference for media richness would be higher for males than females. Females would be more open to adapting to new, leaner media because they would lose very little with fewer cues; this phenomenon would be related to the rich get richer hypothesis [41, 42]. This hypothesis proposes that those who are gifted in communication benefit the most from learning to use new media because it gives them yet another means to communicate with others. This study should shed light on how these two frameworks come into play with media richness preference.

\subsection{H3: Personality Traits Will Predict Media Richness Pref-} erence. The two perspectives mentioned above may come into play. The rich get richer hypothesis [41] would suggest that people with traits predicting better communication would prefer the leaner media because it gives them yet another tool to do what they do well, with little loss from having fewer cues. The social compensation hypothesis [40] would suggest that people with the traits that predict weaker communication skills would prefer the leaner media because these new media are less threatening and easier to manage. It is not easy to predict which traits would predict the most effective communication across the various media. However, high extraversion, high agreeableness, high conscientiousness, high openness to experience, and low neuroticism have all been linked to many socially desirable qualities. These five traits predict higher self-esteem, locus of control, and generalized self-efficacy [43]. They also predict emotional intelligence as measured by several scales [44] along with well-being and satisfaction with life [45]. Thus it is possible that extraversion, agreeableness, conscientiousness, openness to experience, and low neuroticism will either positively predict media richness preference (the social compensation hypothesis) or negatively predict media richness preference (the rich get richer preference). This study will shed light on which framework is most relevant for the individual differences examined.

\section{Method}

Diverse participants with at least basic skills in computer mediated communication participated in an online survey about their media preferences which were then analyzed through correlational and regression analyses. The survey included measures of personality traits, preferences for various media, and demographic information.

4.1. Participants. The target population consisted of adults at least 18 years old who had at least a minimal competency with computer mediated communication (e.g., the ability to email or access a web site). A convenience sample was recruited from the authors' social networks and email distribution lists. A total of 299 people participated in the survey and produced usable results. Twenty-seven surveys were only partially completed (age was most commonly omitted). Thus $N$ varied from 272 to 299 for the various analyses.

4.2. Measures. The survey included three sections: personality traits, communication preferences, and demographic information.

The traits described by the five-factor model of personality were measured by a modified short inventory developed and validated by Rammstedt and John [46]. This inventory 
TABLE 2: Descriptive statistics and bivariate correlations.

\begin{tabular}{|c|c|c|c|c|c|c|c|c|}
\hline & E & A & $\mathrm{C}$ & $\mathrm{N}$ & $\mathrm{O}$ & Age & Gender & MRP \\
\hline Extraversion & .705 & .106 & $.202^{* *}$ & $-.229^{* *}$ & $.268^{* *}$ & $.187^{* *}$ & $.130^{*}$ & $.183^{* *}$ \\
\hline Agreeableness & & .448 & $.221^{* *}$ & $-.146^{*}$ & .113 & .068 & $.121^{*}$ & $.146^{*}$ \\
\hline Conscientiousness & & & .538 & -.087 & $.226^{* *}$ & $.338^{* *}$ & $.163^{* *}$ & .079 \\
\hline Neuroticism & & & & .466 & $-.184^{* *}$ & -.094 & $.305^{* *}$ & $-.134^{*}$ \\
\hline Openness & & & & & .641 & -.103 & .046 & $.121^{*}$ \\
\hline Age & & & & & & - & .049 & -.005 \\
\hline Gender & & & & & & & - & $-.137^{*}$ \\
\hline Media richness preference & & & & & & & & .593 \\
\hline$N$ & 299 & 299 & 299 & 299 & 299 & 272 & 285 & 295 \\
\hline Mean & 4.11 & 5.19 & 5.01 & 3.57 & 5.47 & 38.3 & 1.51 & .019 \\
\hline $\mathrm{SD}$ & 1.49 & 1.00 & 1.28 & 1.36 & 1.03 & 16.4 & .50 & .57 \\
\hline Scale range or measure & $1-6$ & $1-6$ & $1-6$ & $1-6$ & $1-6$ & Years & $\begin{array}{c}1=\text { male } \\
2=\text { female }\end{array}$ & Mean of $z$-scores \\
\hline
\end{tabular}

Note: E: extraversion; A: agreeableness; C: conscientiousness; N: neuroticism; O: openness; MRP: media richness preference. Cronbach's $\alpha$ is presented along the diagonal.

${ }^{*} p<.05,2$ tailed. ${ }^{* *} p<.01,2$ tailed.

sufficiently predicts the results of longer personality questionnaires, such as the Big Five Inventory [46]. This inventory is especially useful when longer questionnaires are not practical because they would discourage participation [47], as was likely the case in this study. The items measuring extraversion (e.g., "I see myself as someone who is outgoing, sociable"), agreeableness (e.g., "I see myself as someone who is considerate and kind to almost everyone"), conscientiousness (e.g., "I see myself as someone who does a thorough job"), and neuroticism (e.g., "I see myself as someone who gets nervous easily") were measured with the two or three Likert items chosen by Rammstedt and John [46]. Three new items were used to measure openness to experience (e.g. "I see myself as someone who is open to new experiences") because a previous study [48], with a sample similar to that of this study, used the items recommended by Rammstedt and John [46] but found a nonnormal distribution in the responses to the openness items, perhaps indicating miscomprehension; these new items were chosen to be easier to understand. The means, standard deviations, and alphas for the five traits are found in Table 2. It should be noted that the alphas are relatively low because the measures only have two or three items.

To assess the degree to which a participant prefers richer media, participants were presented with a series of questions asking them to indicate their preference for two media. They were asked to respond to Likert items in a form similar to Thurstone's [26] method of paired comparison: "In general, I prefer communicating face to face more than by phone" or "In general, I prefer to communicate by email more than by texting." The response choices ranged from 1: "Disagree strongly" to 7: "Agree strongly." Items that asked for a person's preference for a richer media compared to a leaner media (e.g., "face to face more than by phone") were positively scored and those that asked for a person's preference for a leaner media compared to a richer media (e.g., "by emailing more than by texting") were reverse scored. The media compared are listed in order of richest to leanest in Table 1.
Not all participants used all five media examined in this study. It was assumed, since the survey was administered via the Internet, that all participants communicated using at least three media: face-to-face communication, telephone, and email. Additional questions were asked if they used texting and instant messaging (IM). Based on the media used by each participant, participants were then asked to indicate the strength of their preference for one medium over another in all two-way combinations of the media that they used (e.g., to indicate the strength of their agreement or disagreement to "In general, I prefer communicating by e-mail more than face-to-face," reverse scored). Those who used only the three primary media were asked to make three comparisons $(n=$ 77). Those who used four media (the primary three plus texting, $n=60$, or primary three plus instant messaging, $n=$ 33 ) were asked to make six comparisons, and those who used all five media $(n=128)$ were asked to make ten comparisons. The average participant used four media.

For each comparison, the scores were normalized (after reverse scoring if the item asked about a preference for leaner media compared to richer media). For each participant, the media richness preference was calculated by averaging the normalized scores for each comparison made. Because the normalized scores for each participant were weighted according to the number of comparisons made, the average media richness preference was not zero as would be expected when calculating an average of $z$-scores $(\mathrm{M}=.019, \mathrm{SD}=.575)$. Cronbach's alpha for media richness preference was lower than desired $(\alpha=.593)$ but was reasonably acceptable for an exploratory study [49]. This is probably due to comparisons being made on criteria other than media richness; these criteria would likely include familiarity with a given media, perceived skill in using it, or the permanence of the record left by its use. Comments from several participants also indicated that the telephone differed from the other media because they felt it was intrusive, and thus these participants had a strong preference to avoid it. 
Participants responded to questions about their age, gender, education, and continent where they lived. The range of age was 16 to 84 years. The median age and education were 33 years and 16 years, respectively. The participants were divided equally between the sexes and $89 \%$ lived in North America (Canada and USA); 5\% lived in Europe and 3\% in Australia/Pacific Ocean. There was no relationship between age and gender $(r=.049, p=.421)$ or education and gender $(r$ $=-.071, p=.236)$. Age and education were moderately related $(r=.394, p<.001)$ possibly because many of the younger participants were still in school.

In general, personality traits were correlated to gender and age in the expected directions (see Table 2) [50, 51]. Agreeableness and conscientiousness went up with age while neuroticism and openness went down with age, although not all the correlations were significant. Contrary to most studies, extraversion was positively correlated to age. Agreeableness, conscientiousness, and neuroticism were all higher in females than in males as expected; similarly there was little difference in openness. Females were significantly more extraverted than males, a response often not found [50].

\section{Results}

5.1. Age and Media Richness Preference. Hypothesis 1 was not supported. No significant relationship was found between age and media richness preference $(r=-.006, n=272$, and $p=$ .919; all probabilities are 2-tailed). Although the number of media used decreases with age $(r=-.493, p<.001)$, age does not predict media richness preference, nor does the number of media used $(r=-.064, p=.274)$. The sample size had power $=.700$ to detect a correlation of .15 between age and media richness preference.

5.2. Gender and Media Richness Preference. Hypothesis 2 was supported. Gender significantly predicts media richness preference; specifically, males have a higher preference for media richness than females $(t=2.33, p=.021, n=285$, and $d=-.277$ ). Males have a stronger preference for using richer media to communicate with others, such as face-toface dialog and telephone, than females.

5.3. Personality and Media Richness Preference. Hypothesis 3 was supported (see Table 2). Personality significantly predicts media richness preference; specifically, higher extraversion $(r=.183, p=.002$, and $n=295)$, higher agreeableness $(r=$ $.145, p=.012$, and $n=295)$, higher openness $(r=.122, p=$ .036 , and $n=295)$, and lower neuroticism $(r=-.134, p$ $=.022$, and $n=295$ ) all predict higher media richness preference. Conscientiousness does not significantly predict media richness preference $(r=.079, p=.177$, and $n=295)$.

5.4. Regression Analyses. A two-step regression analysis was performed. The first model predicted media richness preference from gender and age; this model was not significant $\left(R^{2}\right.$ $=.015, p=.131$; see Table 3 ), although the coefficient $\beta$ for gender was significant.
TABLE 3: Regression analyses.

\begin{tabular}{|c|c|c|c|c|c|}
\hline Model & & $R^{2}$ & $\beta$ & $t$ & $p$ \\
\hline \multirow{3}{*}{1} & & .015 & & & .131 \\
\hline & Gender & & $-.123^{*}$ & -2.022 & .044 \\
\hline & Age & & .000 & -.003 & .997 \\
\hline \multirow{7}{*}{2} & & $.077^{* *}$ & & & .001 \\
\hline & Gender & & $-.162^{*}$ & -2.506 & .013 \\
\hline & Age & & -.028 & -.453 & .651 \\
\hline & Extraversion & & $.155^{*}$ & 2.403 & .017 \\
\hline & Agreeableness & & $.144^{*}$ & 2.380 & .018 \\
\hline & Neuroticism & & -.014 & -.215 & .830 \\
\hline & Openness & & .081 & 1.288 & .199 \\
\hline
\end{tabular}

Note: $n=272 .{ }^{*} p<.05 .{ }^{* *} p<.01$. For gender, male $=1$, female $=2$.

In the second model, the four personality traits that had significant zero-order correlations with media richness preference (extraversion, agreeableness, neuroticism, and openness) were added. Both the model (see Table 3 ) and the change in the multiple correlation coefficient are significant $\left(\Delta R^{2}=.062, p=.002\right)$. The coefficients $\beta$ for gender, extraversion, and agreeableness are significant. This model accounts for $7.7 \%$ of the variance in media richness preference in spite of the low coefficients of reliability.

\section{Discussion}

The purpose of this exploratory study was to examine how age, gender, and personality traits influence a person's preference for media richness. Can such information indicate if a person is likely to have a strong preference for rich media such as face-to-face communication or if the person is likely to feel more comfortable with leaner media, such as texting or email?

No evidence was found that age influences a person's preference for media richness. Contrary to the popular idea that young people prefer communicating in more impersonal, leaner media, we found no evidence for such a difference. Although there are differences across gender and associated with personality traits, the old and the young have the same average preference for media richness. Younger people tend to use more means of electronic communication, but this does not influence their preference for the richer media compared to the leaner. Thus the decision to email or meet with a person face to face should be made considering a multitude of factors (message content, recipient skills, sender skills, social context, gender, personality, etc.), but age in itself is not one of the factors that needs to be taken into consideration.

The Main Effect of Gender Was Significant. Males have a higher preference for media richness than females. This can be understood at two levels. First, males are less skilled in communication than are females [35, 37-39]. Thus for an equal amount of information to be communicated in a given period of time, men need a richer media. Males are less efficient in communication processes due to a greater loss 
of information. The richer media allow for greater amounts of information to be communicated quickly, allowing for a loss of information to have less serious consequences. Richer media also allow for immediate elaboration or clarification when necessary. Women have fewer losses of information, so the leaner media are less risky for them. The leaner media may be more convenient, less time consuming, and more conducive to multitasking. Since women have a relatively lower risk of miscommunication, their preference for rich media is lower than men's. This lends support to the "rich get richer" perspective [41, 42]: those who are gifted in communication benefit the most from using new, leaner media because it gives them yet another means to efficiently communicate with others.

A second reason that men may have a higher media richness preference concerns gender differences in communication styles [31,32]. Men tend to be agentic and goal oriented in their communication. The purpose of their communication tends to be the exchange of new, precise information. Any error in communication distracts them from accomplishing the goal. Thus, to reduce the likelihood of a loss of information, men have a greater preference for richer media in which the greater number of cues and synchronicity minimize the likelihood of misinterpretation errors. Women tend to be more communal and expressive in their communication, developing relationships and supporting the person with whom they are communicating. This requires the communication of less new information; the bandwidth of information communicated may be narrower but perfectly appropriate. Thus leaner media may be quite aptly suited for this type of communication. For example, the person who sends hundreds of text messages per day to maintain friendships is quite likely to be communicating the same message to multiple recipients, most of whom may have relatively well defined expectations of what the message will contain; using lean media in such situations carries little risk of information loss.

In the final model of the regression analyses, extraversion and agreeableness both significantly predicted a higher preference for media richness. The strongest predictor of media richness preference was extraversion. Extraverted people tend to be sociable, outgoing, and communicative. Introverted people tend to be reserved, calm, and quiet. Karemaker [29] had found evidence that extraversion predicted a preference for richer media, but the results were only significant for people low in neuroticism. This study has demonstrated that extraversion is a predictor of media richness preference even when controlling for neuroticism. This may be interpreted as indicating that the richness of an interaction, rather than the quantity of interactions, is what lies behind the extravert's motivation to be social, outgoing, and communicative. For the introvert, the extra cues provided by the richer media are less attractive. The motivations that lead them to be reserved, calm, and quiet also lead them towards the leaner media which, by their nature, can be more easily monitored and used in calm and quiet ways.
The second personality trait to predict a higher preference for richer media is agreeableness. Agreeable people are motivated to be warm, caring, and soft-hearted. These traits often manifest themselves in emotions expressing concern. The richer media, which can communicate facial, corporal, and/or tonal cues, are quite appropriate for communicating emotions. Text based communications require greater effort and skill to convey emotional content as well as the richer media. Thus agreeable people have a higher preference for richer media than those who are less agreeable.

The findings concerning both extraversion and agreeableness support the social compensation hypothesis [40, 42]. Those who are weaker in communication skills profit more from computer mediated communication than those who are more gifted.

\section{Conclusion and Implications}

The purpose of this exploratory study was to examine the role that age, gender, and personality traits can play in predicting media richness preference. We can conclude that people who are more extraverted or more agreeable have a higher preference for rich media. Men tend to have a higher preference for rich media than women. Age appears to have little or no relationship with media richness preference.

These differences in media richness preference can be explained by differences in communication styles of males and females and by the greater skill in communication associated with higher extraversion and agreeableness. Men tend to be more agentic and goal oriented in their communication while women tend to be more communal and relationship oriented. This orientation makes rich communication media more attractive to males since it decreases the likelihood of loss of task related information. Lower extraversion and lower agreeableness make leaner, computer mediated communication more attractive because these media limit the rate of exchange of interpersonal information and lessen the need for emotional sensitivity and responsiveness.

On the practical side, this information can be used by managers, employees, friends, and family to help them choose the media they use to communicate. If the situation (including message content, recipient skills, sender skills, and social context) does not provide sufficient information to determine what communication medium would be most appropriate, the results of this study may be useful. By choosing a medium that best corresponds to a recipient's media richness preference, the sender can make it more likely that the message will be understood and dealt with appropriately.

Although significant predictors of the construct were found, much more research needs to be done. The construct of media richness preference needs to be improved. We measured media richness preference by asking each participant 3 to 10 questions about his or her media preferences. The coefficient of reliability for this scale was only marginally sufficient. The reliability might be able to be increased by asking participants to respond to more items. However, it is possible, and likely, that much more than media richness influences 
an individual's preference for one medium over another. Thus future research would benefit from the development of new ways of measuring media richness preference.

This study also used very short measures of personality traits. These scales also had low coefficients of reliability. Because the alphas were low for both variables in each correlation computed, it is quite possible that the relationships detected are actually much stronger than the results indicated. Further research should be done using more reliable personality instruments that measure not just the Big Five traits, but also more specific traits (such as the facets of the Big Five) which might provide more information on how personality is related to media richness preference and how these traits interact with gender and age.

\section{Conflict of Interests}

The authors declare that there is no conflict of interests regarding the publication of this paper.

\section{Acknowledgment}

This paper was supported by Konkuk University.

\section{References}

[1] R. L. Daft and R. H. Lengel, "Information richness. A new approach to managerial behavior and organization design," Research in Organizational Behavior, vol. 6, pp. 191-233, 1984.

[2] R. L. Daft and R. H. Lengel, "Organizational information requirements, media richness and structural design," Management Science, vol. 32, no. 5, pp. 554-571, 1986.

[3] A. Ramirez, J. Dimmick, J. Feaster, and S. F. Lin, "Revisiting interpersonal media competition the gratification niches of instant messaging, e-mail, and the telephone," Communication Research, vol. 35, no. 4, pp. 529-547, 2008.

[4] A. Puce, "Perception of nonverbal cues," in The Oxford Handbook of Cognitive Neuroscience, K. Ochsner and S. M. Kosslyn, Eds., vol. 2, pp. 148-164, Oxford Press, Oxford, UK, 2013.

[5] J. D’Ambra, R. E. Rice, and M. O'Connor, “Computer-mediated communication and media preference: an investigation of the dimensionality of perceived task equivocality and media richness," Behaviour and Information Technology, vol. 17, no. 3, pp. 164-174, 1998.

[6] A. Tversky and D. Kahneman, "Rational choice and the framing of decisions," Journal of Business, vol. 59, no. 4, pp. 251-278, 1986.

[7] J. Fulk, J. Schmitz, and C. W. Steinfield, "A social influence model of technology use," in Organizations and Communication Technology, J. Fulk and C. W. Steinfield, Eds., pp. 117-140, Sage, Newbury Park, Calif, USA, 1990.

[8] L. P. Robert and A. R. Dennis, "Paradox of richness: a cognitive model of media choice," IEEE Transactions on Professional Communication, vol. 48, no. 1, pp. 10-21, 2005.

[9] R. E. Petty and J. T. Cacioppo, Communication and Persuasion: Central and Peripheral Routes to Attitude Change, Springer, New York, NY, USA, 1986.

[10] J. Webster and L. K. Trevino, "Rational and social theories as complementary explanations of communication media choices: two policy-capturing studies," Academy of Management Journal, vol. 38, pp. 1544-1572, 1995.
[11] A. Bandura, Social Learning Theory, Prentice Hall, Englewood Cliffs, NJ, USA, 1977.

[12] J. Short, E. Williams, and B. Christie, The Social Psychology of Telecommunications, John Wiley \& Sons, New York, NY, USA, 1976.

[13] J. B. Walther, "Computer-mediated communication: impersonal, interpersonal, and hyperpersonal interaction," Communication Research, vol. 23, no. 1, pp. 3-43, 1996.

[14] B. D. Minsky and D. B. Marin, "Why faculty members use e-mail: the role of individual differences in channel choice," Journal of Business Communication, vol. 36, no. 2, pp. 194-211, 1999.

[15] D. P. McAdams, "What do we know when we know a person?" Journal of Personality, vol. 63, no. 3, pp. 365-396, 1995.

[16] D. C. McClelland, "How motives, skills, and values determine what people do," American Psychologist, vol. 40, no. 7, pp. 812825, 1985.

[17] D. G. Winter, O. P. John, A. J. Stewart, E. C. Klohnen, and L. E. Duncan, "Traits and motives: toward an integration of two traditions in personality research," Psychological Review, vol. 105, no. 2, pp. 230-250, 1998.

[18] P. T. Costa, Jr, and R. R. McCrae, Revised NEO Personality Inventory (NEO-PI-R) and NEO Five-Factor Inventory (NEOFFI), Psychological Assessment Resources, Odessa, Fla, USA, 1992.

[19] R. R. McCrae and P. T. Costa Jr., Personality in Adulthood, Guilford Press, New York, NY, USA, 2nd edition, 2003.

[20] O. P. John and S. Srivastava, "The big-five trait taxonomy: history, measurement, and theoretical perspectives," in Handbook of Personality: Theory and Research, L. A. Pervin and O. P. John, Eds., pp. 102-138, Guilford Press, New York, NY, USA, 1999.

[21] R. B. Zajonc, "Feeling and thinking: preferences need no inferences," American Psychologist, vol. 35, no. 2, pp. 151-175, 1980.

[22] R. B. Zajonc, "On the primacy of affect," American Psychologist, vol. 39, no. 2, pp. 117-123, 1984.

[23] P. T. Costa Jr. and R. R. McCrae, "From catalog to classification: Murray's needs and the five-factor model," Journal of Personality and Social Psychology, vol. 55, no. 2, pp. 258-265, 1988.

[24] H. Stumpf, "The factor structure of the Personality Research Form: a cross-national evaluation," Journal of Personality, vol. 61, no. 1, pp. 27-48, 1993.

[25] L. Kelly and J. A. Keaten, "Development of the affect for communication channels scale," Journal of Communication, vol. 57, no. 2, pp. 349-365, 2007.

[26] L. L. Thurstone, "The method of paired comparisons for social values," Journal of Abnormal and Social Psychology, vol. 21, no. 4, pp. 384-400, 1927.

[27] J. C. McCroskey, A. D. Heisel, and V. P. Richmond, "Eysenck's big three and communication traits: three correlational studies," Communication Monographs, vol. 68, no. 4, pp. 360-366, 2001.

[28] D. J. Reid and F. J. M. Reid, Insights into the Social and Psychological Effects of Text Messaging, University of Plymouth, Plymouth, UK, 2004.

[29] D. Karemaker, Face to Face or Mediated Communication? Personality Makes a Difference, University of Amsterdam, Amsterdam, The Netherlands, 2005.

[30] R. Junco and J. Mastrodicasa, Connecting to The Net Generation: What Higher Education Professionals Need to Know About Today's Students, NASPA, Washington, DC, USA, 2007. 
[31] A. E. Abele, "The dynamics of masculine-agentic and femininecommunal traits: findings from a prospective study, Journal of Personality and Social Psychology, vol. 85, no. 4, pp. 768-776, 2003.

[32] D. Bakan, The Duality of Human Existence: An Essay on Psychology and Religion, Rand McNally, Chicago, Ill, USA, 1966.

[33] M. D. Kirtley and J. B. I. Weaver, "Exploring the impact of gender role self-perception on communication style," Women's Studies in Communication, vol. 22, no. 2, pp. 190-209, 1999.

[34] K. J. Prager, The Psychology of Intimacy, Guilford Publications, 1995.

[35] J. J. Gross and O. P. John, "Mapping the domain of expressivity: multimethod evidence for a hierarchical model," Journal of Personality and Social Psychology, vol. 74, no. 1, pp. 170-191, 1998.

[36] V. L. DeFrancisco, "The sounds of silence: how men silence women in marital relations," Discourse \& Society, vol. 2, no. 4, pp. 413-423, 1991.

[37] J. A. Hall and D. Matsumoto, "Gender differences in judgments of multiple emotions from facial expressions," Emotion, vol. 4, no. 2, pp. 201-206, 2004.

[38] W. J. Ickes, Everyday Mind Reading: Understanding What Other People Think and Feel, Prometheus Books, Amherst, NY, USA, 2003.

[39] J. A. Hall, Nonverbal Sex Differences: Accuracy of Communication and Expressive Style, Johns Hopkins University Press, Baltimore, Md, USA, 1990.

[40] K. Y. A. McKenna and J. A. Bargh, "Plan 9 from cyberspace: the implications of the internet for personality and social psychology," Personality and Social Psychology Review, vol. 4, no. 1, pp. 57-75, 2000.

[41] R. Kraut, S. Kiesler, B. Boneva, J. Cummings, V. Helgeson, and A. Crawford, "Internet paradox revisited," Journal of Social Issues, vol. 58, no. 1, pp. 49-74, 2002.

[42] P. M. Valkenburg and J. Peter, "Preadolescents' and adolescents' online communication and their closeness to friends," Developmental Psychology, vol. 43, no. 2, pp. 267-277, 2007.

[43] T. A. Judge, A. Erez, J. E. Bono, and C. J. Thoresen, "Are measures of self-esteem, neuroticism, locus of control, and generalized self-efficacy indicators of a common core construct?" Journal of Personality and Social Psychology, vol. 83, no. 3, pp. 693-710, 2002.

[44] M. A. Brackett and J. D. Mayer, "Convergent, discriminant, and incremental validity of competing measures of emotional intelligence," Personality and Social Psychology Bulletin, vol. 29, no. 9, pp. 1147-1158, 2003.

[45] J. Musek, "A general factor of personality: evidence for the Big One in the five-factor model," Journal of Research in Personality, vol. 41, no. 6, pp. 1213-1233, 2007.

[46] B. Rammstedt and O. P. John, "Measuring personality in one minute or less: a 10-item short version of the Big Five inventory in English and German," Journal of Research in Personality, vol. 41, no. 1, pp. 203-212, 2007.

[47] M. B. Donnellan, F. L. Oswald, B. M. Baird, and R. E. Lucas, "The mini-IPIP scales: tiny-yet-effective measures of the big five factors of personality," Psychological Assessment, vol. 18, no. 2, pp. 192-203, 2006.

[48] D. Dunaetz, Personality and Conflict Style: Effects on Membership Duration in Voluntary Associations, Lambert Academic Press, Saarbrücken, Germany, 2011.

[49] J. C. Nunnally and I. H. Bernstein, Psychometric Theory, McGraw-Hill, New York, NY, USA, 3rd edition, 1994.
[50] A. Feingold, "Gender differences in personality: a metaanalysis," Psychological Bulletin, vol. 116, no. 3, pp. 429-456, 1994.

[51] R. R. McCrae, P. T. Costa Jr., L. M. Pedroso et al., "Age differences in personality across the adult life span: parallels in five cultures," Developmental Psychology, vol. 35, no. 2, pp. 466$477,1999$. 

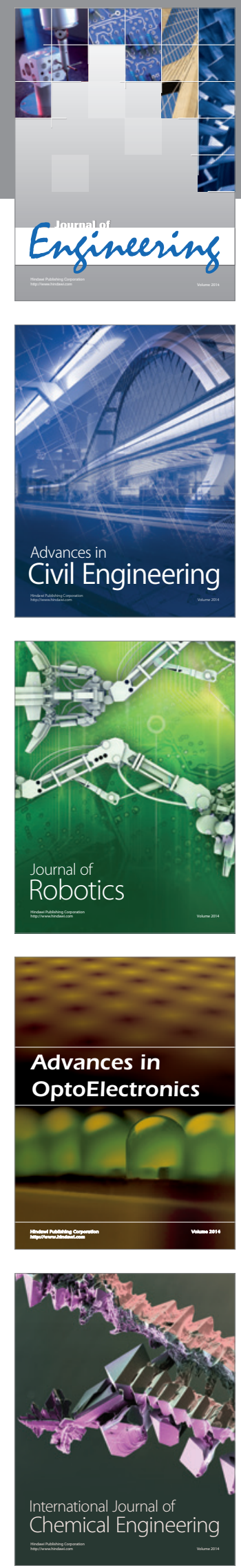

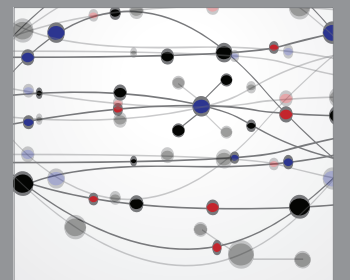

The Scientific World Journal
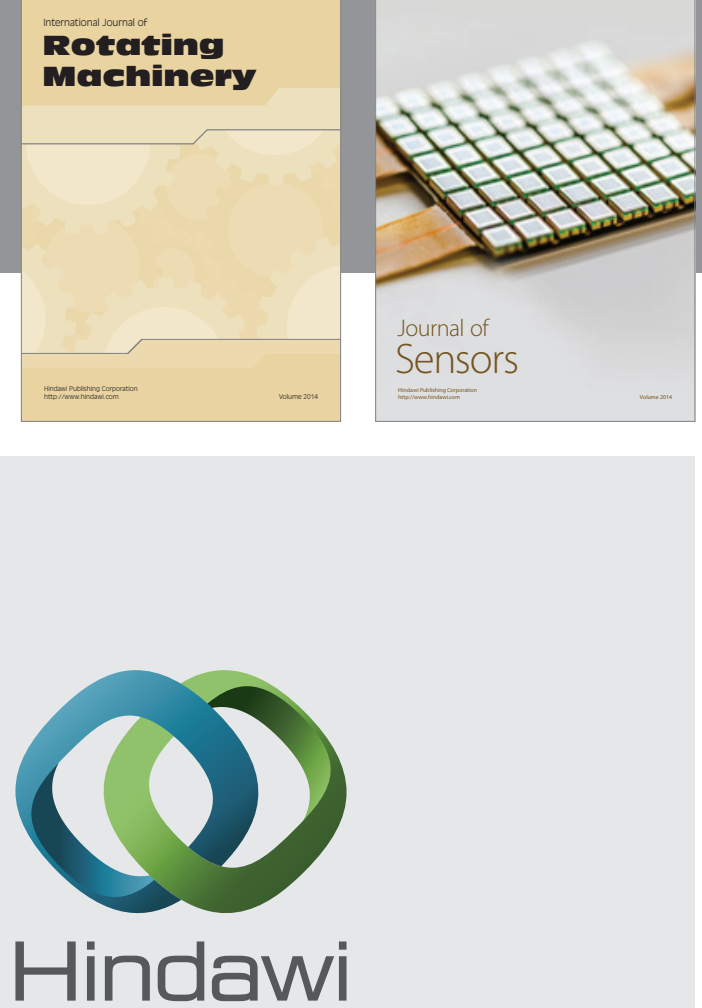

Submit your manuscripts at http://www.hindawi.com
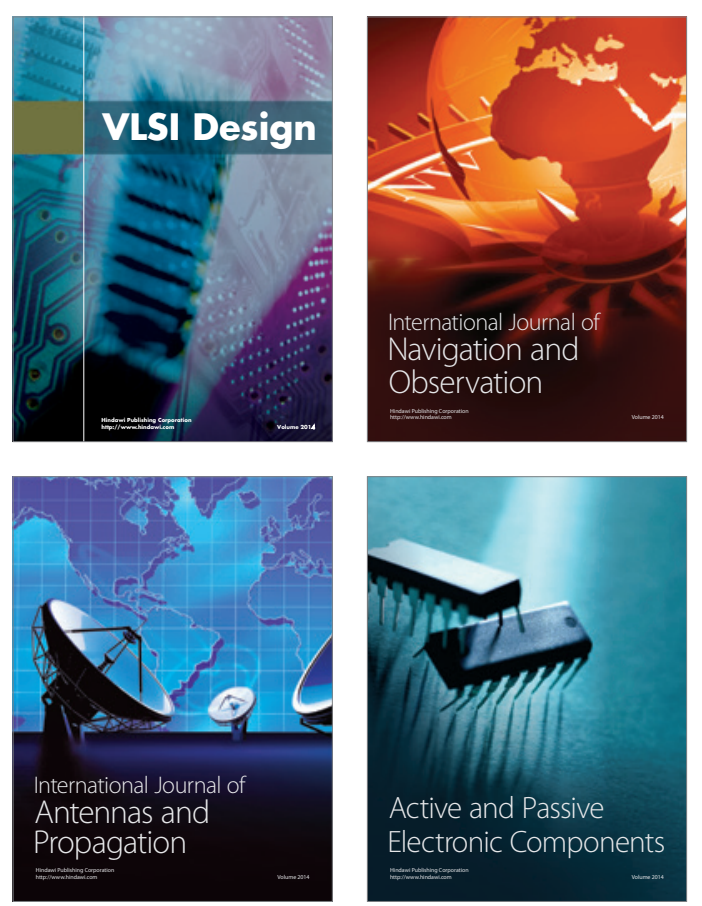
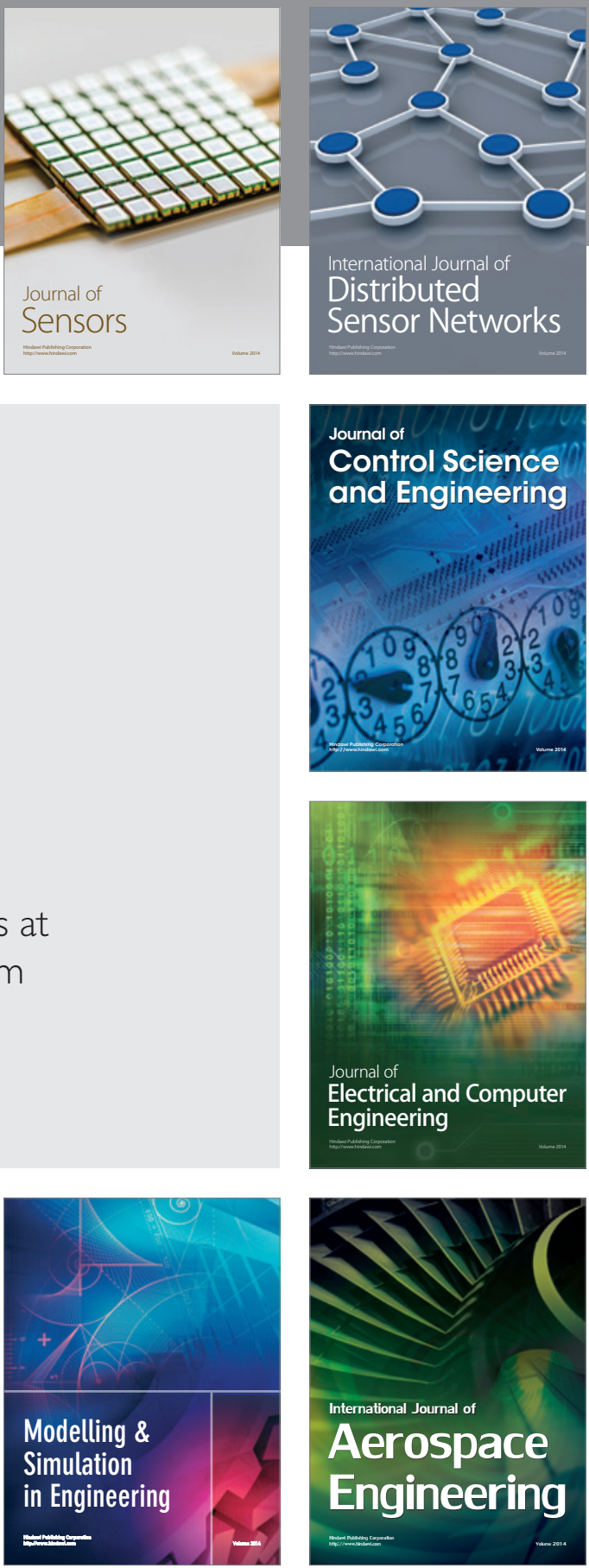

Journal of

Control Science

and Engineering
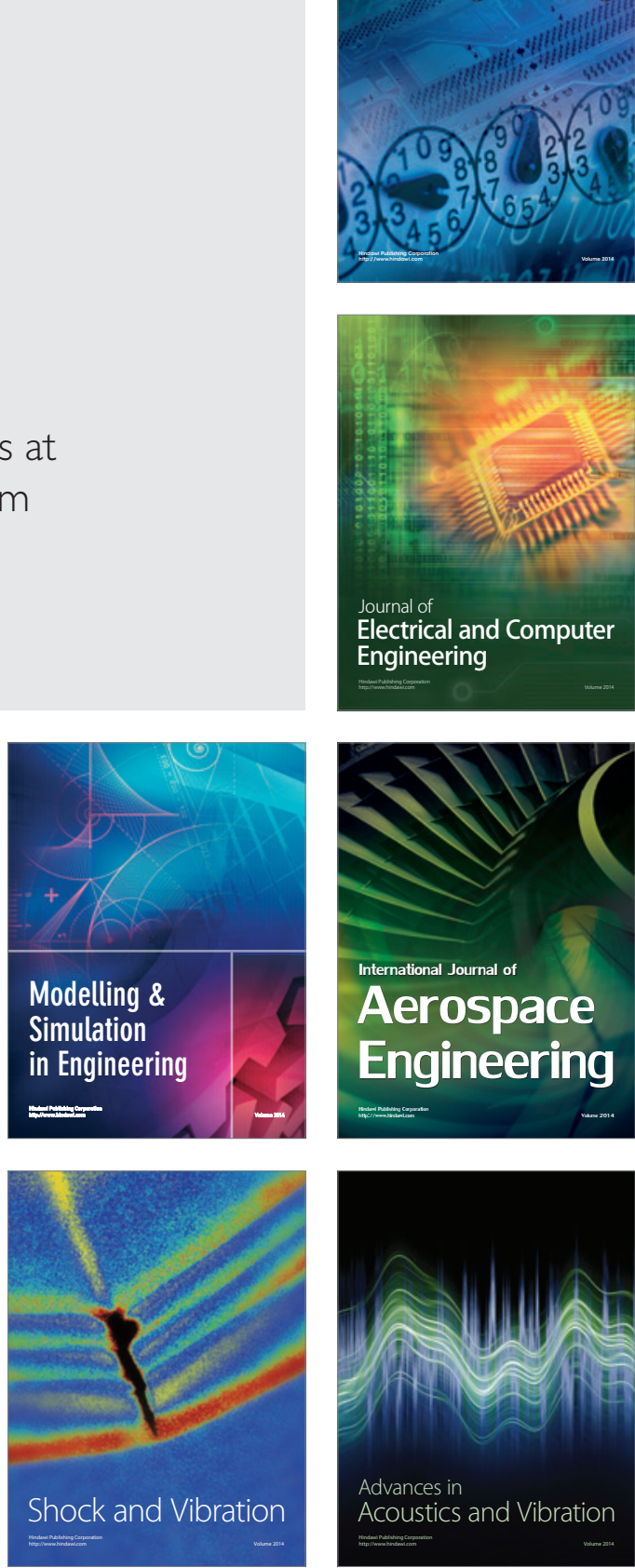